\title{
AXIN1 protects against testicular germ cell tumors via the PI3K/AKT/mTOR signaling pathway
}

\author{
HAILIANG XU ${ }^{1}$, YUNYUN FENG ${ }^{2}$, ZHANKUI JIA $^{3}$, JINJIAN YANG $^{3}$, XUEREN LU $^{1}$, JUN LI $^{1}$, \\ MINGLIANG XIA $^{1}$, CHUNRU WU $^{4}$, YONGGANG ZHANG ${ }^{5}$ and JIANHUA CHEN ${ }^{6}$ \\ Departments of ${ }^{1}$ Urinary Surgery and ${ }^{2}$ Pediatrics, The Zhumadian City Center Hospital, Zhumadian, \\ Henan 463000; ${ }^{3}$ Department of Urinary Surgery, The First Affiliated Hospital of Zhengzhou University, \\ Zhengzhou, Henan 450000; Departments of ${ }^{4}$ Gynecology, ${ }^{5}$ Emergency and ${ }^{6}$ General Surgery, \\ The Zhumadian City Center Hospital, Zhumadian, Henan 463000, P.R. China
}

Received September 21, 2015; Accepted February 27, 2017

DOI: 10.3892/ol.2017.6214

\begin{abstract}
Axis inhibition protein 1 (AXIN1) is characterized as a tumor suppressor in numerous types of cancer. However, the functional role of AXIN1 in the testicular germ cell tumors (TGCTs) remains unclear. The human embryonal carcinoma-derived cell line NTera 2 was transfected with a recombinant AXIN1 expression vector (pcDNA3.1-AXIN1) and/or a small interfering RNA (siRNA) directed against AXIN1 (siAXIN). Following transfection, the mRNA and protein levels of AXIN1 were determined via reverse transcription-quantitative polymerase chain reaction analysis and western blotting, respectively. In addition, cell viability, apoptosis and the expression of apoptosis-associated proteins [apoptosis regulator $\mathrm{Bax}(\mathrm{Bax})$ and $\mathrm{B}$-cell lymphoma (Bcl)-2] and phosphatidylinositol 3-kinase (PI3K)/protein kinase $\mathrm{B}$ $(\mathrm{AKT}) / \mathrm{mammalian}$ target of rapamycin (mTOR) signaling pathway proteins [phosphorylated (p)-mTOR, mTOR, p-AKT, AKT, P-70S ribosomal protein S6 (S6) and S6] were assessed. AXIN1 mRNA and protein levels were increased following transfection with pcDNA3.1-AXIN1 and decreased following transfection with siAXIN1 compared with their respective control groups. After overexpression of AXIN1, NTera2 cell viability and expression of Bcl-2, p-mTOR p-AKT and p-S6 protein was decreased, while apoptosis and Bax protein levels were increased, compared with the control group. However, there was no significant difference in AXIN1 mRNA expression, apoptosis or Bax/Bcl-2 protein expression when NTera 2 cells were simultaneously transfected with pcDNA3.1-AXIN1+siAXIN1. In conclusion, the results of the present study indicate that overexpression of AXIN1 protects against TGCTs via inhibiting the PI3K/AKT/mTOR signaling
\end{abstract}

Correspondence to: Dr Hailiang $\mathrm{Xu}$, Department of Urinary Surgery, The Zhumadian City Center Hospital, 747 Zhonghua Road, Zhumadian, Henan 463000, P.R. China

E-mail: xuhailiang5588@126.com

Key words: axis inhibition protein 1, testicular germ cell tumors, $\mathrm{PI} 3 \mathrm{~K} / \mathrm{AKT} / \mathrm{mTOR}$ signaling pathway pathway, suggesting that AXIN1 may be a potential target for gene therapy in TGCTs.

\section{Introduction}

Testicular germ cell tumors (TGCTs) are relatively rare, representing $\sim 1-1.5 \%$ of all malignancies diagnosed in males and $\sim 5 \%$ of urological tumors in males in general (1). However, TGCTs are the most frequently observed solid tumor among men aged between 15 and 35 years old (2). Additionally, it has been reported that the incidence of TGCTs has increased over the last 30-40 years (3). Despite this, the 5-year disease-free survival of patients with TGCTs is the highest amongst any other solid malignancy in males due to effective modern therapy, including platinum-based combination chemotherapy regimens, and close surveillance following surgery. In addition, the cure rate for patients with TGCTs is $95-96 \%(4,5)$. However, $\sim 5 \%$ of patients with TGCTs still develop treatment resistance (6). Therefore, a better understanding of the pathogenesis of TGCTs is important in order to develop novel treatments.

The functional role of axis inhibition protein 1 (AXIN1), a multi-domain scaffold protein, has been associated with the tumorigenesis and progression of a number of diseases, including hepatitis B virus-related hepatocellular carcinoma and gastrointestinal cancer (7-10). AXIN1 acts as a tumor suppressor, and thus mutations of AXIN1 serve a significant role in carcinogenesis $(8,11)$. Additionally, in combination with several different protein complexes AXIN1 has been reported to be involved in the Wnt, transforming growth factor (TGF)- $\beta$, stress activated protein kinase JNK1 (JNK) and cellular tumor antigen p53 (p53) signaling pathways (12-15). Furthermore, activation of the Wnt/ $\beta$-catenin signaling pathway in human germ cell tumors has been reported (16), a cancer that AXINI mutation has been associated with (17). However, little information is available regarding the association between the functional role of AXIN1 and the phosphatidylinositol 3-kinase (PI3K)/protein kinase B (AKT)/mammalian target of rapamycin (mTOR) signaling pathway in TGCTs.

The aim of the present study was to determine the functional role of AXIN1 in TGCTs. Furthermore, whether AXIN1 functions through the PI3K/AKT/mTOR signaling 
pathway was investigated. The results of the current study may contribute to the identification and development of novel drugs for the treatment of TGCTs.

\section{Materials and methods}

Cell lines and culture. The human embryonal carcinoma (EC)-derived cell line NTera2 was purchased from the Shanghai Institute of Biochemistry and Cell Biology of the Chinese Academy of Sciences (Shanghai, China). Cells were maintained in Dulbecco's modified Eagle's medium (Sigma; Merck KGaA, Darmstadt, Germany) supplemented with $10 \%$ fetal calf serum (BD Biosciences, San Jose, CA, USA), $58.5 \mathrm{mg} / \mathrm{ml} \mathrm{L}$-Glutamine (Gibco), $100 \mathrm{U} / \mathrm{ml}$ penicillin and $100 \mathrm{mg} / \mathrm{ml}$ streptomycin (all from Thermo Fisher Scientific, Inc., Waltham, MA, USA) at $37^{\circ} \mathrm{C}$ in an incubator with $5 \% \mathrm{CO}_{2}$.

Transfection. When the cells reached $70 \%$ confluence, they were subjected to transfection with small interfering RNA (siRNA) or an expression construct using Lipofectamine ${ }^{\circledR} 2000$ reagent $\left(1 \times 10^{6}\right.$ cells/well) (Invitrogen; Thermo Fisher Scientific, Inc.) following the manufacturer's protocol. AXIN1 was knocked down using a siRNA (siAXIN1) and a scrambled siRNA (siControl) was used as a control. The sequence of siRNAs was listed as following: siAXIN1, 5'GGGAUAAGCCUGUUCAGGATT3', and siControl, 5'AAAATCGACTCGTTTTTGCTC3'. The target sequences were designed and constructed by Shanghai GenePharma Co., Ltd. (Shanghai, China). In addition, an AXIN1 expression vector (pcDNA3.1-AXIN1) was constructed and confirmed by sequencing (7). As a control, the empty construct pcDNA3.1 was transfected into NTera 2 cells. For the cells simultaneously transfected with siAXIN1 and pcDNA3.1-AXIN1, the cells simultaneously transfected with scrambled siRNA and empty construct were considered as a control.

MTT cell viability assay. The survival of NTera2 cells was determined using the MTT colorimetric assay. Briefly, cells were collected, washed with PBS seeded into a 96-well plate $\left(1 \times 10^{6}\right.$ cells/well) and incubated at $37^{\circ} \mathrm{C}$. After $24,48,72$ and $96 \mathrm{~h}$ incubation, $10 \mu 1$ MTT was added to each well, and the cells were cultured for a further $4 \mathrm{~h}$ at $37^{\circ} \mathrm{C}$. The absorbance at $595 \mathrm{~nm}$ was measured with a Synergy ${ }^{\mathrm{TM}} 4$ Hybrid Microplate Reader (BioTek Instruments, Inc., Winooski, VT, USA).

Apoptosis assay. Cell apoptosis was assessed using an Annexin V-fluorescein isothiocyanate (FITC) Apoptosis Detection kit (R\&D Systems, Inc., Minneapolis, MN, USA) according to the manufacturer's protocol. Briefly, the cells were harvested $\left(2 \times 10^{5}\right.$ cells/well), washed with PBS and re-suspended with binding buffer. Subsequently, the mixture was incubated with $5 \mu 1$ Annexin V-FITC and $5 \mu 1$ propidium iodide (PI). After incubation at room temperature in the dark for $15 \mathrm{~min}$, the cells were analyzed with a flow cytometer (BD LSR Flow Cytometer; BD Biosciences). The collected data were analyzed using CellQuest ${ }^{\mathrm{TM}}$ software (version 3.0; BD Biosciences).

Reverse transcription-quantitative polymerase chain reaction (RT-qPCR) analysis. AXIN1 mRNA levels in NTera2 cells transfected with pcDNA3.1-AXIN1 or siAXIN1 for $48 \mathrm{~h}$ was determined using RT-qPCR analysis. Total RNA was extracted with the TRIzol reagent (Invitrogen; Thermo Fisher Scientific, Inc.) according to the manufacturer's protocol. First strand $5 \mu \mathrm{l}$ complementary (c)DNA was subjected to real-time PCR reaction using an ABI PRISM 7700 System and TaqMan reagents (Applied Biosystems; Thermo Fisher Scientific, Inc.) according to the manufacturer's protocol (18). GAPDH mRNA was used as a reference. The sequence of mRNA was listed as following: AXIN1; forward, 5'AACACATGGTCATGCCAAGC3'; reverse, 5'TTCTCAGCGTCCTCTGTGG3' (19); and GAPDH; forward, 5'TCCTGCACCACCAACTGCTTAG3'; reverse, 5'AGTGGC AGTGATGGCATGGACT3'. The PCR thermocycler conditions included: initial denaturation was performed at $96^{\circ} \mathrm{C}$ for $15 \mathrm{sec}$, followed by 30 cycles of denaturation at $97^{\circ} \mathrm{C}$ for $15 \mathrm{sec}$, annealing at $62^{\circ} \mathrm{C}$ for $5 \mathrm{sec}$, extension at $72^{\circ} \mathrm{C}$ for $50 \mathrm{sec}$ and the final extension was at $72^{\circ} \mathrm{C}$ for $10 \mathrm{~min}$. Gene expression was quantified using the $2^{-\Delta \Delta \mathrm{Cq}}$ method (20).

Western blotting. A total of $24 \mathrm{~h}$ after transfection, protein was extracted from the cells by using a radioimmunoprecipitation lysis extraction kit (Thermo Fisher Scientific, Inc.). The protein concentration was measured using a BCA Protein Assay kit (Pierce; Thermo Fisher Scientific, Inc.) according to the manufacturer's protocol. A total of $20 \mu \mathrm{g}$ per lane of protein samples were resolved on a $12 \%$ gel using SDS-PAGE and blotted onto polyvinylidene difluoride membranes. The resulting membranes were blocked in $5 \%$ milk in $0.1 \%$ Tris-buffered saline-Tween for $2 \mathrm{~h}$. Subsequently, the membranes were treated with the following primary monoclonal antibodies overnight at $4^{\circ} \mathrm{C}$ : Anti-AXIN1 (cat. no. ab131372; dilution, 1:1,000; Abcam, Cambridge, UK), anti-apoptosis regulator Bax (Bax) (cat. no. sc-65,532; dilution, 1:1,000), anti-B-cell lymphoma (Bcl)-2 (cat. no. sc-509; dilution, 1:1,000), anti-phosphorylated (p)-mTOR (cat. no. sc-293132; dilution, 1:1,000), anti-mTOR (cat. no. sc-400140; dilution, 1:1,000), anti-p-AKT (cat. no. sc-7985-R; dilution, 1:1,000), anti-AKT (cat. no. sc-24500; dilution, 1:1,000), anti-P-70S ribosomal protein S6 (S6) (cat. no. sc-8416; dilution, 1:1,000), or anti-S6 (cat. no. sc-9027; dilution, 1:1,000). GAPDH (cat. no. sc-293335; dilution, $1: 1,000)$ was used as the internal control. Then the appropriate goat anti-rabbit IgG (cat. no. sc-2004; dilution, 1:10,000) or goat anti-mouse IgG (cat. no. sc-2005; dilution, 1:10,000) were used for $2 \mathrm{~h}$ at room temperature. All antibodies were purchased from Santa Cruz Biotechnology, Inc. (Dallas, TX, USA) except anti-AXIN1. Immunoreactive protein bands were detected by enhanced chemiluminescence western blotting substrate (Pierce; Thermo Fischer Scientific, Inc.).

Statistical analysis. All experiments were performed $\geq 2$ times. The data are expressed as the mean \pm standard deviation. Data analysis was carried out SPSS software (version 16.0; SPSS, Inc., Chicago, IL, USA). One-way analysis of variance followed by Tukey post hoc test was performed to calculate the P-values. $\mathrm{P}<0.05$ was considered to indicate a statistically significant difference.

\section{Results}

Confirming the results of siRNA and construct transfection on AXIN1 expression. To explore the effects of transfection on 


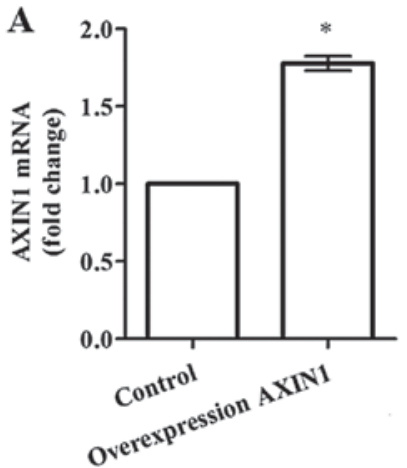

$\mathbf{D}$

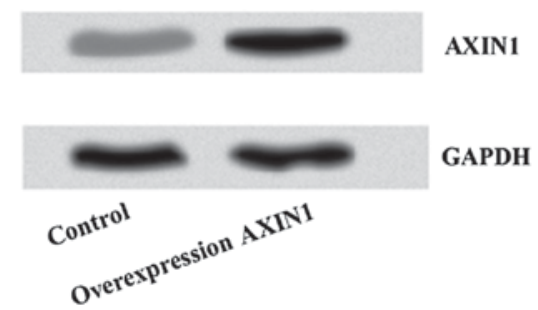

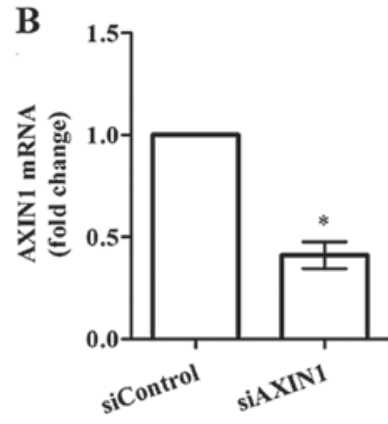

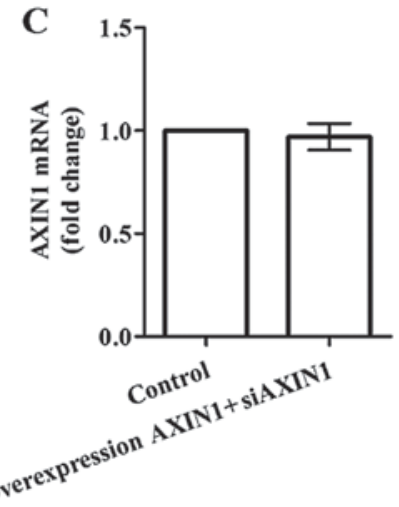

$\mathbf{E}$

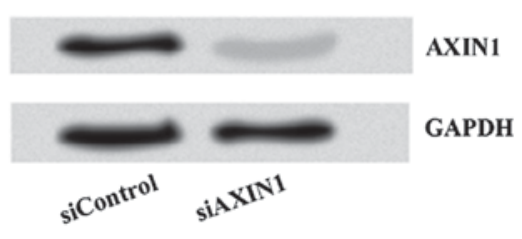

Figure 1. Effects of siAXIN1 and pcDNA3.1-AXIN1 transfection on AXIN1 mRNA and protein expression in NTera2 cells. Relative expression of AXIN1 mRNA after transfection with (A) pcDNA3.1-AXIN1, (B) siAXIN1 and (C) pcDNA3.1-AXIN1+siAXIN1. Expression of AXIN1 protein after transfection with (D) pcDNA3.1-AXIN1 and (E) siAXIN1. *P<0.05 vs. Control/siControl groups. AXIN1, axis inhibition protein 1; siAXIN1, siRNA targeting AXIN1; siControl, control siRNA.

AXIN1 expression, an AXIN1 expression vector (pcDNA3.1-AXIN1) and AXIN1-specific siRNA were constructed. As illustrated in Fig. 1A, the mRNA expression of AXIN1 was significantly increased following transfection with pcDNA3.1-AXIN1 compared with the control group $(\mathrm{P}<0.05)$. By contrast, the mRNA expression levels of AXIN1 were significantly lower in the siAXIN1 group compared with the siControl group $(\mathrm{P}<0.05$; Fig. 1B). However, no significant difference was observed in AXIN1 mRNA expression following simultaneous transfection with pcDNA3.1-AXIN1+siAXIN1 compared with the control group (Fig. 1C). Western blots for the protein level of AXIN1 revealed similar results (Fig. 1D and E).

Effect of AXIN1 overexpression and silencing on TGCT cell viability. To explore the effects of AXIN1 overexpression and silencing on TGCT cell viability, an MTT assay was performed. A total of $24 \mathrm{~h}$ after transfection, cell viability was significantly increased by in the group transfected with siAXIN1 and markedly decreased in the group transfected with pcDNA3.1-AXIN1 compared with the control group of cells simultaneously transfected with scrambled siRNA and empty construct (both $\mathrm{P}<0.05$; Fig. 2), indicating that AXIN1 acts as a tumor suppressor in TGCTs.

Effect of AXIN1 overexpression and silencing on TGCT cell apoptosis. Flow cytometry was performed to explore the effects of AXIN1 overexpression and silencing on TGCT cell apoptosis (Fig. 3). There was no significant difference in the percentage of apoptosis between the siAXIN1 (Fig. 3B) or pcDNA3.1-AXIN1+siAXIN1 (Fig. 3C) groups compared with their respective controls. However, the percentage of apoptosis

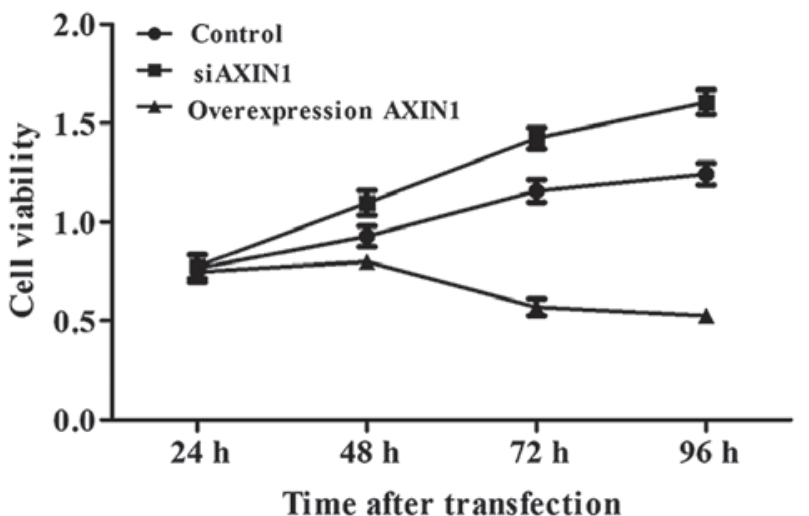

Figure 2. Effect of siAXIN1 and pcDNA3.1-AXIN1 transfection on NTera2 cell viability. AXIN1, axis inhibition protein 1; siAXIN1, siRNA targeting AXIN1.

was significantly increased in the pcDNA3.1-AXIN1 group compared with the control group (Fig. 3A; $\mathrm{P}<0.05$ ), suggesting that overexpression of AXIN1 induces TGCT cell apoptosis.

Effect of AXIN1 overexpression and silencing on the expression of Bax and Bcl-2 protein in TGCT cells. To explore the potential of AXIN1 overexpression-induced TGCT cell apoptosis, the levels of the apoptosis-associated proteins Bax and Bcl-2 in NTera2 cells transfected with siAXIN1 and/or pcDNA3.1-AXIN1 were measured. This revealed that the expression of Bax was markedly increased and the expression of Bcl-2 was markedly decreased after transfection with pcDNA3.1-AXIN1 compared with the control group (Fig. 4A). However, there was no notable difference in 
A

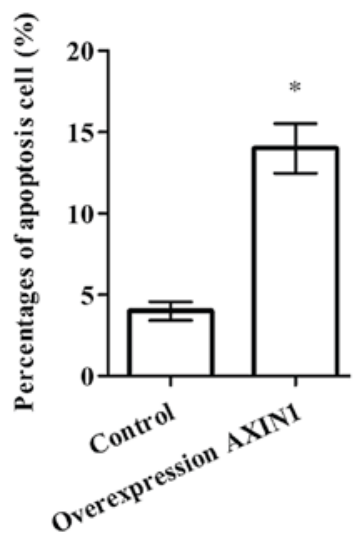

D
B

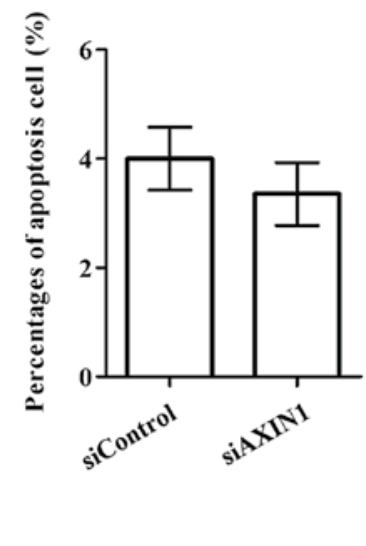

C

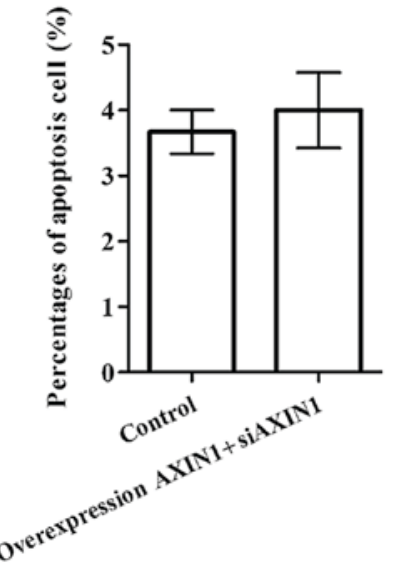

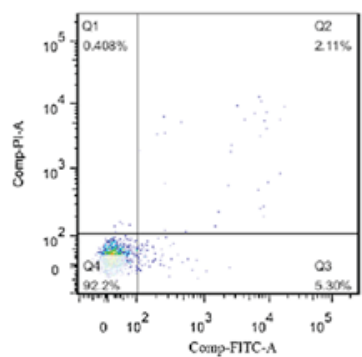

$\mathbf{E}$

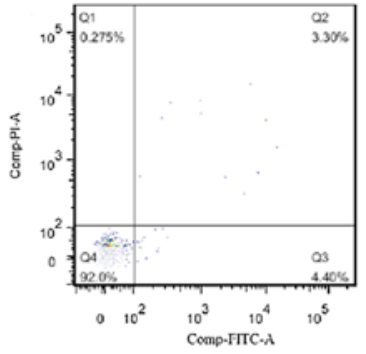

siControl

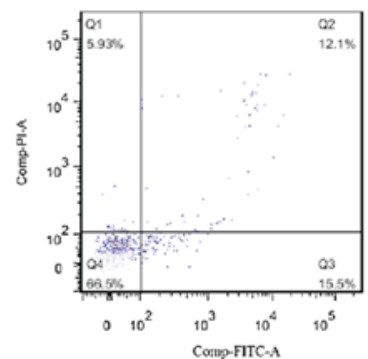

Overexpression AXIN1

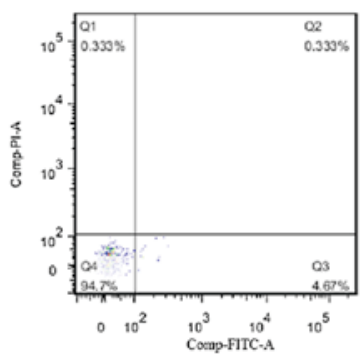

siAXIN1

Figure 3. Effect of siAXIN1 and pcDNA3.1-AXIN1 transfection on NTera2 cell apoptosis. Percentages of apoptotic cells after transfection with (A) pcDNA3.1-AXIN1, (B) siAXIN1 and (C) pcDNA3.1-AXIN1+siAXIN1. Images of flow cytometry analysis after transfection with (D) pcDNA3.1-AXIN1 and (E) siAXIN1. AXIN1, axis inhibition protein 1; siAXIN1, siRNA targeting AXIN1; siControl, control siRNA.

A

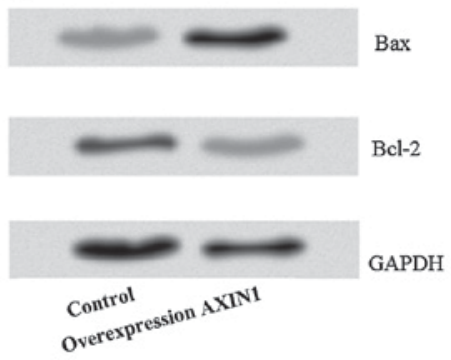

B
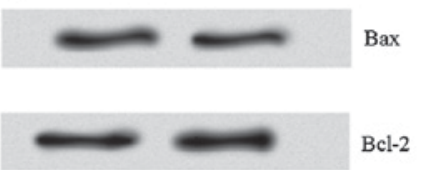

cl-2

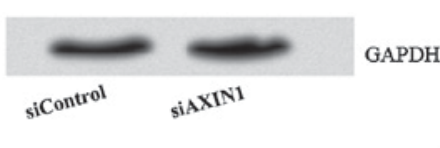

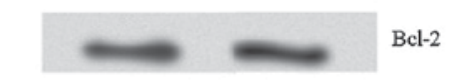

$\mathrm{C}$

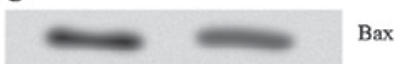
Bax

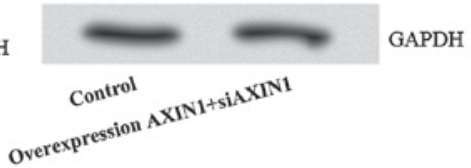

Figure 4. Effect of siAXIN1 and pcDNA3.1-AXIN1 transfection on NTera2 cell expression of Bax and Bcl-2 protein. Expression of Bax and Bcl-2 protein after transfection with (A) pcDNA3.1-AXIN1, (B) siAXIN1 and (C) pcDNA3.1-AXIN1+siAXIN1. AXIN1, axis inhibition protein 1; Bax, apoptosis regulator Bax; Bcl-2, B-cell lymphoma-2; siAXIN1, siRNA targeting AXIN1; siControl, control siRNA.

the protein levels of Bax or Bcl-2 in the siAXIN1 (Fig. 4B) or pcDNA3.1-AXIN1+siAXIN1 (Fig. 4C) groups compared with their respective controls.
Effect of AXIN1 overexpression and silencing on the PI3K/AKT/mTOR signaling pathway in TGCT cells. To investigate the potential signaling pathway through which 


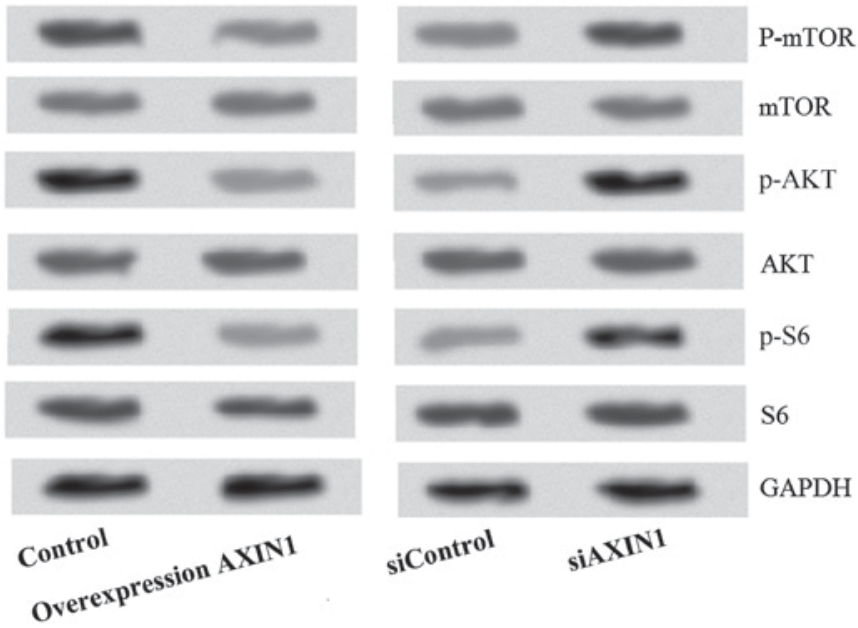

Figure 5. Effect of siAXIN1 and pcDNA3.1-AXIN1 transfection on the $\mathrm{PI} 3 \mathrm{~K} / \mathrm{AKT} / \mathrm{mTOR}$ signaling pathway in NTera 2 cells. AXIN1, axis inhibition protein 1; PI3K, phosphatidylinositol 3-kinase; AKT, protein kinase B; mTOR, mammalian target of rapamycin; S6, 70S ribosomal protein S6; p-, phosphorylated; siAXIN1, siRNA targeting AXIN1.

AXIN1 overexpression was inducing TGCT cell apoptosis, the levels of $\mathrm{PI} 3 \mathrm{~K} / \mathrm{AKT} / \mathrm{mTOR}$ signaling pathway proteins (p-mTOR, mTOR, p-AKT, AKT, p-S6 and S6) was investigated (Fig. 5). The results demonstrated that the protein levels of p-AKT, P-mTOR and p-S6 were markedly reduced following transfection with pcDNA3.1-AXIN1, indicating that the PI3K/AKT/mTOR signaling pathway is inhibited by AXIN1 overexpression.

\section{Discussion}

In the present study, AXIN1 was confirmed to be a candidate tumor suppressor gene in TGCTs. Overexpression of AXIN1 could inhibit TGCT cell viability and induce human EC-derived NTera2 cell apoptosis through increasing the expression of proapoptotic Bax protein, while decreasing the expression of antiapoptotic Bcl-2 protein. The PI3K/Akt/mTOR signaling pathway was also demonstrated to serve an important role in AXIN1 overexpression-induced TGCT cell apoptosis. These results suggest that $A X I N 1$ is a potential target for gene therapy in TGCTs.

AXIN1 was previously identified to be a product of the mouse Fused locus, and is mapped to human chromosome $16 \mathrm{p} 13.3$ with $87 \%$ similarity to the mouse protein (21). AXIN1 is associated with regulating axis formation during embryonic development (21). Two naturally occurring splicing variants of AXIN1, variant 1 (AXIN1V1) and variant 2 (AXIN1V2) have been reported. AXIN1V1 encodes an 862-amino acid (AA)-long polypeptide, whereas AXIN1V2 is a shorter form of AXIN that lacks 36 AAs from exon 8 (8). AXIN1 serves as a scaffold protein through interacting with numerous proteins, enabling it to facilitate the degradation $\beta$-catenin, which suggests that it has a tumor suppressor function (22). Several signaling pathways, including that of Wnt, TGF- $\beta$, JNK1 and p53, have been reported to be associated with AXIN1 (23-25). A previous study suggested that AXIN1 mutation may be associated with germ cell tumors (17). However, there is little information with respect to the effect of AXIN1 overexpression, rather than mutation, on TGCTs.

One of the mechanisms underlying tumorigenesis is the activation of essential cellular signaling pathways. The essential and well-studied PI3K/AKT/mTOR signaling pathway serves important roles in tumorigenesis $(26,27)$. This signaling pathway contributes to cell proliferation, differentiation, metabolism, cytoskeletal reorganization and apoptosis (28). Activation of the PI3K/AKT/mTOR signaling pathway promotes cancer cell survival and therapy resistance (29-31). Following activation of the PI3K/AKT/mTOR signaling pathway by membrane tyrosine kinase growth factor receptors or $\mathrm{G}$ protein-coupled receptors $(32,33)$, functional $\mathrm{PI} 3 \mathrm{~K}$ is translocated to the plasma membrane where it causes the phosphorylation of phosphatidylinositol 3,4,5-triphosphate (PIP3) (33). Thereafter, p-PIP3 recruits 3'-phosphoinositide-dependent kinase 1 (PDK1) and AKT (34), leading to the phosphorylation and activation of AKT. Phosphorylation of AKT increases cell survival by inactivating proapoptotic factors, including $\operatorname{Bax}(34,35)$. Subsequently, p-AKT activates several downstream targets, including those in the mTOR signaling pathway. Activation of mTOR leads to an increase in protein synthesis, including that of certain proteins that are associated with the pathogenesis of a number of tumors, such as cyclin D1 (36). Additionally, activated mTOR can directly phosphorylate PDK1 and activate ribosomal protein S6 kinase b-1 ( $\left.\mathrm{P} 70^{\mathrm{S} 6 \mathrm{~K}}\right)$. P70 ${ }^{\mathrm{S} 6 \mathrm{~K}}$ initiates the ribosomal translation of mRNA into proteins that is essential for cell growth, progression and metabolism by phosphorylating S6. Thus, activation of PI3K/AKT/mTOR signaling pathway is a key event in tumorigenesis.

Since AXIN1 may act as a tumor suppressor in TGCTs, the theory that this this protective effect may be through inhibition of the PI3K/AKT/mTOR signaling pathway was investigated. To confirm this hypothesis, transfection techniques were utilized to dysregulate the expression of AXIN1 in NTera2 cells. The effects of transfection on AXIN1 expression were confirmed by RT-qPCR and western blotting. The effects of AXIN1 dysregulation on NTera2 cell viability and apoptosis were then examined. The results showed that NTera2 cell viability was significantly increased by knockdown of AXIN1 and markedly decreased by overexpression of AXIN1, further suggesting that AXIN1 functions as a tumor suppressor in TGCTs. In addition, the percentage of apoptotic cells was significantly increased by overexpression of AXIN1. This result was in line with those of previous studies, which also suggested that AXIN1 could significantly induce cancer cell apoptosis $(11,37)$. Next, the potential mechanism of AXIN1 overexpression-induced apoptosis was explored. Overexpression of AXIN1 markedly increased Bax protein expression and markedly decreased $\mathrm{Bcl}-2$ protein expression. One explanation for this effect is that AXIN1 acts of AKT to inhibit the PI3K/AKT/mTOR signaling pathway, decreasing apoptosis-associated protein expression and apoptosis.

The levels of PI3K/AKT/mTOR signaling pathway proteins were assessed in the present study. This demonstrated that the protein levels of p-AKT, p-mTOR and p-S6 were markedly reduced by overexpression of AXIN1, indicating that the PI3K/AKT/mTOR signaling pathway is inhibited by overexpression of AXIN1. Arnold et al (38) suggested that AXIN1 could negatively regulate proto-oncogene c-Myc 
protein expression at a post-translational level, thus acting a tumor suppressor (38). However, in the present study it remains unclear whether AXIN1 regulates or coordinates with oncoproteins to exert antitumor activity.

In conclusion, the results of the present study confirm that AXIN1 is a candidate tumor suppressor gene in TGCTs and indicate that it exerts this effect through inhibiting the $\mathrm{PI} 3 \mathrm{~K} / \mathrm{AKT} / \mathrm{mTOR}$ signaling pathway. This suggests that AXIN1 is a potential target for gene therapy in TGCTs. However, further research is required to determine the mechanism through which AXIN1 regulates the PI3K/AKT/mTOR signaling pathway in cancer.

\section{References}

1. Chia VM, Quraishi SM, Devesa SS, Purdue MP, Cook MB and McGlynn KA: International trends in the incidence of testicular cancer, 1973-2002. Cancer Epidemiol Biomarkers Prev 19: $1151-1159,2010$.

2. Vasdev N, Moon A and Thorpe AC: Classification, epidemiology and therapies for testicular germ cell tumours. Int J Dev Biol 57 133-139, 2013.

3. Purdue MP, Devesa SS, Sigurdson AJ and McGlynn KA: International patterns and trends in testis cancer incidence. Int $\mathrm{J}$ Cancer 115: 822-827, 2005.

4. Ries LAG, Young JL Jr, Keel GE, Eisner MP, Lin YD and Horner M-JD (eds): SEER Survival Monograph: Cancer Survival Among Adults: U.S. SEER Program, 1988-2001, Patient and Tumor Characteristics. National Cancer Institute, SEER Program, NIH Pub. No. 07-6215, Bethesda, MD, 2007.

5. Hayes-Lattin B and Nichols CR: Testicular cancer: A prototypic tumor of young adults. Semin Oncol 36: 432-438, 2009.

6. Raghavan D: Testicular cancer: Maintaining the high cure rate. Oncology (Williston Park) 17: 218-229, 234-235, 2003.

7. Li J, Quan H, Liu Q, Si Z, He Z and Qi H: Alterations of axis inhibition protein 1 (AXIN1) in hepatitis B virus-related hepatocellular carcinoma and overexpression of AXIN1 induces apoptosis in hepatocellular cancer cells. Oncol Res 20: 281-288, 2013.

8. Salahshor S and Woodgett JR: The links between axin and carcinogenesis. J Clin Pathol 58: 225-236, 2005.

9. Park JY, Park WS, Nam SW, Kim SY, Lee SH, Yoo NJ, Lee JY and Park CK: Mutations of beta-catenin and AXIN I genes are a late event in human hepatocellular carcinogenesis. Liver Int 25 70-76, 2005.

10. Mazzoni SM and Fearon ER: AXIN1 and AXIN2 variants in gastrointestinal cancers. Cancer Lett 355: 1-8, 2014.

11. Satoh S, Daigo Y, Furukawa Y, Kato T, Miwa N, Nishiwaki T, Kawasoe T, Ishiguro H, Fujita M, Tokino T, et al: AXIN1 mutations in hepatocellular carcinomas and growth suppression in cancer cells by virus-mediated transfer of AXIN1. Nat Genet 24 245-250, 2000.

12. Kishida S, Yamamoto H, Ikeda S, Kishida M, Sakamoto I, Koyama S and Kikuchi A: Axin, a negative regulator of the wnt signaling pathway, directly interacts with adenomatous polyposis coli and regulates the stabilization of beta-catenin J Biol Chem 273: 10823-10826, 1998.

13. Deng F, Li S, Xu W, Zou Z, Ke Z and Zeng F: AXIN1-related CSRNP1 mRNA expression and its transcriptional regulation in TGF-beta1-induced tumor cells. Nan Fang Yi Ke Da Xue Xue Bao 33: 1122-1126, 2013 (In Chinese).

14. Zhang Y, Neo SY, Wang X, Han J and Lin SC: Axin forms a complex with MEKK1 and activates c-Jun NH(2)-terminal kinase/stress-activated protein kinase through domains distinct from Wnt signaling. J Biol Chem 274: 35247-35254, 1999.

15. Rui Y, Xu Z, Lin S, Li Q, Rui H, Luo W, Zhou HM, Cheung PY, $\mathrm{Wu} \mathrm{Z}$, Ye Z, et al: Axin stimulates p53 functions by activation of HIPK2 kinase through multimeric complex formation. EMBO J 23: 4583-4594, 2004.

16. Fritsch MK, Schneider DT, Schuster AE, Murdoch FE and Perlman EJ: Activation of Wnt/beta-catenin signaling in distinct histologic subtypes of human germ cell tumors. Pediatr Dev Pathol 9: 115-131, 2006.
17. Dahmen RP, Koch A, Denkhaus D, Tonn JC, Sörensen N, Berthold F, Behrens J, Birchmeier W, Wiestler OD and Pietsch T: Deletions of AXIN1, a component of the WNT/wingless pathway, in sporadic medulloblastomas. Cancer Res 61: 7039-7043, 2001.

18. Ngalame NN, Tokar EJ, Person RJ and Waalkes MP: Silencing KRAS overexpression in arsenic-transformed prostate epithelial and stem cells partially mitigates malignant phenotype. Toxicol Sci 142: 489-496, 2014.

19. Figeac $\mathrm{N}$ and Zammit PS: Coordinated action of Axin1 and Axin 2 suppresses $\beta$-catenin to regulate muscle stem cell function. Cell Signal 27: 1652-1665, 2015.

20. Livak KJ and Schmittgen TD: Analysis of relative gene expression data using real-time quantitative PCR and the 2(-Delta Delta C(T)) Method. Methods 25: 402-408, 2001.

21. Zeng L, Fagotto F, Zhang T, Hsu W, Vasicek TJ, Perry WL III, Lee JJ, Tilghman SM, Gumbiner BM and Costantini F: The mouse Fused locus encodes Axin, an inhibitor of the Wnt signaling pathway that regulates embryonic axis formation. Cell 90: 181-192, 1997.

22. de la Roche M, Ibrahim AE, Mieszczanek J and Bienz M: LEF1 and B9L shield $\beta$-catenin from inactivation by Axin, desensitizing colorectal cancer cells to tankyrase inhibitors. Cancer Res 74: 1495-1505, 2014.

23. Liu C, Li Y, Semenov M, Han C, Baeg GH, Tan Y, Zhang Z, Lin $\mathrm{X}$ and $\mathrm{He} \mathrm{X}$ : Control of beta-catenin phosphorylation/degradation by a dual-kinase mechanism. Cell 108: 837-847, 2002.

24. L'Esperance S, Popa I, Bachvarova M, Plante M, Patten N, Wu L, Têtu B and Bachvarov D: Gene expression profiling of paired ovarian tumors obtained prior to and following adjuvant chemotherapy: Molecular signatures of chemoresistant tumors. Int J Oncol 29: 5-24, 2006.

25. Rada P, Rojo AI, Offergeld A, Feng GJ, Velasco-Martín JP, González-Sancho JM, Valverde ÁM, Dale T, Regadera J and Cuadrado A: WNT-3A regulates an Axin1/NRF2 complex that regulates antioxidant metabolism in hepatocytes. Antioxid Redox Signal 22: 555-571, 2015.

26. Liu P, Cheng H, Roberts TM and Zhao JJ: Targeting the phosphoinositide 3-kinase pathway in cancer. Nat Rev Drug Discov 8: 627-644, 2009

27. Porta C, Paglino C and Mosca A: Targeting PI3K/Akt/mTOR signaling in cancer. Front Oncol 4: 64, 2014.

28. Polivka J Jr and Janku F: Molecular targets for cancer therapy in the PI3K/AKT/mTOR pathway. Pharmacol Ther 142: 164-175, 2014.

29. Engelman JA: Targeting PI3K signalling in cancer: Opportunities, challenges and limitations. Nat Rev Cancer 9: 550-562, 2009.

30. Burris HA III: Overcoming acquired resistance to anticancer therapy: Focus on the PI3K/AKT/mTOR pathway. Cancer Chemother Pharmacol 71: 829-842, 2013.

31. Cavazzoni A, Bonelli MA, Fumarola C, La Monica S, Airoud K, Bertoni R, Alfieri RR, Galetti M, Tramonti S, Galvani E, et al: Overcoming acquired resistance to letrozole by targeting the PI3K/AKT/mTOR pathway in breast cancer cell clones. Cancer Lett 323: 77-87, 2012.

32. Stern DF: ERBB3/HER3 and ERBB2/HER2 duet in mammary development and breast cancer. J Mammary Gland Biol Neoplasia 13: 215-223, 2008

33. Zhao L and Vogt PK: Class I PI3K in oncogenic cellular transformation. Oncogene 27: 5486-5496, 2008.

34. Cantley LC: The phosphoinositide 3-kinase pathway. Science 296: 1655-1657, 2002.

35. Engelman JA, Luo J and Cantley LC: The evolution of phosphatidylinositol 3-kinases as regulators of growth and metabolism. Nat Rev Genet 7: 606-619, 2006.

36. Grewe M, Gansauge F, Schmid RM, Adler G and Seufferlein T: Regulation of cell growth and cyclin D1 expression by the constitutively active FRAP-p70s6K pathway in human pancreatic cancer cells. Cancer Res 59: 3581-3587, 1999.

37. Biechele TL, Kulikauskas RM, Toroni RA, Lucero OM, Swift RD, James RG, Robin NC, Dawson DW, Moon RT and Chien AJ: Wnt/ $\beta$-catenin signaling and AXIN1 regulate apoptosis triggered by inhibition of the mutant kinase BRAFV600E in human melanoma. Sci Signal 5: ra3, 2012.

38. Arnold HK, Zhang X, Daniel CJ, Tibbitts D, Escamilla-Powers J, Farrell A, Tokarz S, Morgan C and Sears RC: The Axin1 scaffold protein promotes formation of a degradation complex for c-Myc. EMBO J 28: 500-512, 2009. 